\title{
Sub-microsecond pulsed pumping as a means of suppressing amplified spontaneous emission in tandem pumped fiber amplifiers
}

\author{
Andrew Malinowski, Jonathan H. V. Price and Michalis N. Zervas, Member, IEEE
}

\begin{abstract}
We propose a new tandem pumping scheme in threelevel rare-earth doped optical fibers where energy storage in a pump fiber laser enables much shorter ( $\sim 500 \mathrm{~ns}$ ) pump pulses than is possible with pulsed diode laser pumping. This has the effect of reducing the time during which the final amplifier has high inversion and hence reduces the ASE when compared to continuous wave (c.w.) tandem pumping. We simulate the gain and ASE dynamics in a pulse-pumped Yb-doped fiber amplifier and show that the ratio of amplified signal to ASE background can be improved by up to 2 orders of magnitude over a large range of pulse energies compared to c.w. tandem pumping. Sub-microsecond pulsed tandem pumping offers a relatively straightforward, as yet experimentally untried, way to substantially reduce undesirable ASE compared to the levels in diode-pumped fiber amplifiers.
\end{abstract}

Index Terms - Pulsed fiber amplifier, tandem pumping

\section{INTRODUCTION}

$\mathrm{F}$ IBER lasers have advantages including of high efficiency and stable long-term performance as well as the ability for significant power-scaling due to their waveguiding character and their nearly ideal geometry for heat dissipation [1]. Driven by manufacturing applications, power of $100 \mathrm{~kW}$ have been demonstrated from c.w. fiber lasers. Pulsed fiber lasers present additional challenges because the high peak powers and tightly confined wave-guide mode result in unwanted nonlinear effects but very large mode rod-type fibers are now routinely achieving $>1 \mathrm{~mJ}$ output at $>100 \mathrm{~W}$ average power levels using the same directly diode-pumped double-clad fiber geometry as used in c.w. lasers. These attractive attributes have led to the proposed use of coherently combined fiber laser arrays for future particle accelerators [2, 3]. Test-bed systems currently envisioned would produce $40 \mathrm{~J}$ pulses at a repetition rate of $10 \mathrm{kHz}$ and an average power of $40 \mathrm{~kW}$ from an Yb-fiber amplifier array. However, the complexity of coherently combining the output in a stable way and the high average power will create tight tolerances on the thermal fluctuations permissible in the

This paragraph of the first footnote will contain the date on which you submitted your paper for review. J. Price was supported by funding from EPSRC (UK) Centre for Innovative Manufacturing in Photonics: $\mathrm{EP} / \mathrm{H} 02607 \mathrm{X} / 1$. The first two authors contributed equally to this paper. The data for this paper can be found at: http://dx.doi.org/10.5258/SOTON/381336

A. Malinowski is with SPI Lasers, 6 Wellington Park, Tollbar Way, Hedge End, Southampton, SO30 2QU, U.K. multiple fiber amplifiers and suggest that work will be necessary to reduce the ASE as this is typically a significant fraction of the output in low pulse-repetition-rate fiber lasers. Motivated by this challenge, we have investigated whether pulsed tandem pumping would reduce the thermal load and ASE for low repetition rate Yb-fiber amplifiers.

Tandem or in-band pumping was developed in the context of Er fiber research [4] but has been used more widely since then for power scaling of $\mathrm{Yb}$ fiber lasers [5]. The practical use of tandem pumping for power scaling has so-far been largely confined to high power c.w. in Yb-fiber systems partly because of the increased complexity. At the $100 \mathrm{~kW}$ power level, even fiber lasers require careful thermal management and technical issues arise such as reliably splicing the large numbers of pump diodes. Tandem-pumping schemes, where one or more fiber lasers pump a fiber amplifier, address both these issues [5-7] and indeed the most powerful fiber lasers realized to date are tandem pumped system $[8,9]$. It enables the majority of the brightness enhancement to be performed in the pump fiber amplifier and balances quantum defect heating across the pump and signal fiber amplifiers.

A challenge for pulsed systems is that the in-band absorption cross-sections in the 1010-1030 nm range are lower than at 975 $\mathrm{nm}$ absorption peak and so efficient pump-absorption requires the device length is increased, which leads to limiting nonlinear effects. A single mode pump source would enable corepumping of the final tandem pumped amplifier. This results in pump absorption at a level where device length is reasonable.

Yb-fiber amplifiers work well when CW pumped if the repetition rate is in the $\mathrm{MHz}$ region as then the time over which energy is stored from the c.w. pump diodes is sub-micro-second and there is only a short time during which the system is highly inverted before the signal pulse extracts the energy and reduces the inversion level. At $10 \mathrm{kHz}$ the c.w. pump diodes would lead to a somewhat gradual energy accumulation and hence there is a longer time between achieving a high inversion and the arrival of the signal pulse that extracts the energy, increasing the average ASE. This is also likely to increase the rate of

J.H.V. Price is with the Optoelectronics Research Centre, University of Southampton, Southampton, SO17 1BJ, U.K.

M.N. Zervas is both a Professor at the Optoelectronics Research Centre and Chief Scientist at SPI Lasers. 
photodarkening. It should be stressed though that a proper estimation of the PD impact should take into account the seed power levels and the resulting average and peak inversions, in addition to the host material properties. This detailed study is beyond the scope of this paper.

Based on our experience testing fiberised 975nm pump diodes with c.w. powers in the range 10-40 $\mathrm{W}$ from a range of manufacturers in a pulsed mode, we have found that such pump diodes can usually be used at low duty cycle at peak powers of 5 to $7 \times$ the c.w. rated output power without long term degradation.

However for a $10 \mathrm{kHz}$ repetition rate, this would only reduce the "on" time from $100 \mu$ s in the c.w. pumped case to $20 \mu$ s in the pulsed case. If tandem pumping is used then optical energy storage in the first amplifier operating at $1018 \mathrm{~nm}$ can be used to accumulate the diode pump energy and this could be output in e.g. $500 \mathrm{~ns}$ pump pulses for the longer wavelength tandem pumped amplifier. Then by creating a high inversion in the final amplifier only in the short period immediately preceding the pulse used for energy extraction, the time available for ASE to build-up is reduced compared to c.w. pumped systems. Therefore pulsed tandem pumping could potentially be a useful approach for future high pulse energy coherently combined fiber laser facilities.

In this paper, we have used numerical simulations to consider whether pulsed tandem can lead to a reduction in the ASE power. We focus on $\mathrm{Yb}$ amplifiers and simulate tandem pumping using a rate equation model [10], which considers signal gain in the amplifier, broadband ASE, background loss and Rayleigh backscattering. As shown below, we find that there is a dramatic reduction in ASE when comparing c.w. and pulsed tandem pumping. As well as uses such as electron acceleration [2], there are a range of other applications for coherently combined fiber amplifier arrays including proposals for space-debris management [11] and scaled-down systems would also be useful for industry.

\section{DESCRIPTION OF THE NUMERICAL MODEL USED IN SIMULATIONS}

The model uses our own code and it calculates an effective mode overlap with the dopant for pump and signal based on the index profile of the fiber. The quasi-three-level $\mathrm{Yb}^{3+}$ system is assumed to have 2 energy manifolds [12], so the model deals with the interaction between a number of signals and a single inverted population. The pump and signal are treated as spectrally narrow, while the ASE is described by a number of channels with frequency bandwidth equal to the frequency spacing between the channels, seeded with the standard photonper-mode assumption. Forward and backward propagating ASE channels are modeled. The only direct interaction between optical modes is back-scattering. The rate equations were solved numerically, using the method of characteristics [13], with a spatial grid of $5 \mathrm{~cm}$, and a time step corresponding to the signals travelling one spatial step. The evolution of the amplifier is modeled over several cycles of pumping, until the results over each cycle converge. This generates information on the start-up dynamics of the amplifier, but only the performance after the amplifier has stabilized will be discussed in this paper.

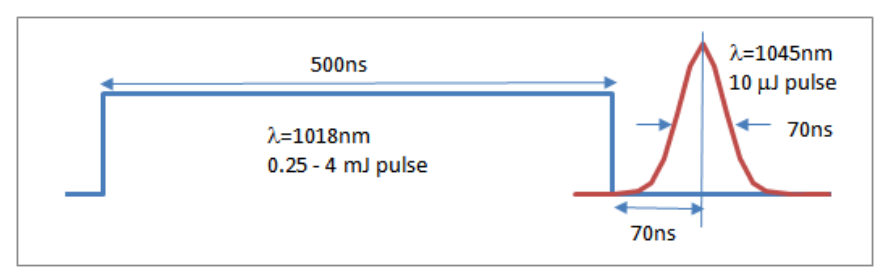

Fig. 1 Schematic of input pump and signal pulses

Initial parameters for the signal and pump powers were chosen using the model to ascertain where the limits on extractable energy were found in a survey of parameter-space. We were motivated by industrial applications, so chose a conventional large-mode-are fiber and ps pulses for the study. The maximum reduction in ASE is seen at rather high pulse energies, so we performed a brief calculation to verify that a pulse format could be selected that kept the peak power below the stimulated-Raman threshold. This led to the choice of a 70 ns envelope for the signal pulse. We focused on ps pulses for which divided pulse amplification (DPA) [14] can scale the energy of a single pulse and high repetition rates enable increases in the average power. The pulse pumping technique is not compatible with evenly spaced $\mathrm{GHz}$ pulse trains. However, it is compatible with burst-mode operation, using widely spaced bunches of ps pulses. This has been shown to improve the speed of material processing compared to the same energy delivered in an evenly spaced pulse train[15]. This mode of operation also has scientific uses such as for photo-injectors in large free-electron systems [16] and has been successfully demonstrated in high performance fiber laser systems [17]. For our chosen example of $70 \mathrm{~ns}$ signal pulses at $10 \mathrm{kHz}$ repetition rate a picosecond pulse burst could be comprised of $700 \times 10 \mu \mathrm{J}$ pulses sliced from a $10 \mathrm{GHz}$ pulse train (100 ps pulse spacing). Then for 10 ps pulses using 8 x DPA in the pulse division stage would give $\sim 80 \%-90 \%$ duty cycle within the packet.

SRS is minimized by pre-shaping the pulses to obtain a flattop profile after the effects of gain-saturation along the fiber. (High energy square pulses are readily obtainable from ytterbium amplifiers by means of pre-shaping the seed pulses $[18,19])$. For the 70 ns pulse (or pulse burst) considered here, and $23 \mathrm{~dB}$ gain in the amplifier so the output pulse energy is 1.5 $\mathrm{mJ}$, and for a flat-top output pulse, the Raman threshold output pulse energy would be $1.5 \mathrm{~mJ}$ using Smith's paper as an estimate [20], and the B-integral would be $\sim 30$ radians.

The simulations assume pump and signal are both launched into the core of the fiber and are co-propagating. Our simulations used 92 ASE channels, equally spaced in frequency and covering the range 1000-1150 nm. Fiber absorption and emission cross-sections were taken from [21, 22]. The repetition rate was $10 \mathrm{kHz}$ and as shown schematically in Fig. 1, the square pump pulse has a 500 ns duration and the Gaussian signal pulse had a duration of 70 ns full-width half-maximum (FWHM). The pump ends 70 ns (i.e. one FWHM) before the signal so there is little overlap. The pump wavelength was chosen to be $1018 \mathrm{~nm}$, as shorter wavelengths will be absorbed more rapidly in the final amplifier and this is approximately the 
shortest laser wavelength straightforwardly obtainable for the envisioned $975 \mathrm{~nm}$ diode-pumped fiber laser that would be the pulsed pump laser. The signal wavelength was chosen to be $1045 \mathrm{~nm}$ after performing some trial simulations (not shown) to assess the approximate wavelength of the gain peak and its variation for different pump power levels. We simulated a $14 \mu \mathrm{m}$, NA 0.1 core fiber $\left(\mathrm{A}_{\text {eff }}=130 \mu \mathrm{m}^{2}\right)$ with a single transverse mode $\left(\mathrm{LP}_{01}\right)$. The Ytterbium doping concentration was $4.4 \times 10^{26} / \mathrm{m}^{3}$, which is an acceptable value for phosphosilicate host glasses which are more resistant to clustering and photo-darkening than alumino-silicate hosts. The majority of the simulations consider a fiber length of $2 \mathrm{~m}$.

The scattering loss value used in the simulations is about an order of magnitude larger than calculated from for the Rayleigh backscattering in a silica fiber of NA 0.1 [21, 23] [22]. This difference is based on our experimental data for fibers of this type, and is accounted for by known sources of distributed scattering in active fibers [24]. For the optimised $2 \mathrm{~m}$ fiber length, the effects of backscatter were found to be negligible even at this high level. A value for background loss typical of a commercial fiber was chosen $(10 \mathrm{~dB} / \mathrm{km})$ and the strength of the back-scattering $\left(4 \times 10^{-3} \mathrm{~dB} / \mathrm{km}\right)$ was based on our measured values for fibers of similar composition.

We chose operating parameters in the saturated gain regime for efficient energy extraction. The saturation energy at the chosen operating wavelength of $1045 \mathrm{~nm}$ was $130 \mathrm{uJ}$ so after some preliminary simulations we found that aiming to extract energies either in a single pulse or closely spaced pulse-burst of the order of $2 \mathrm{~mJ}$ would illustrate the difference between $\mathrm{CW}$ and pulsed pumping clearly and would require a reasonable final amplifier gain of approximately $\sim 24 \mathrm{~dB}$. For all of the simulations, the average seed power was fixed at $100 \mathrm{~mW}$ (10 $\mu \mathrm{J}$ pulse energy). Launched pump power was varied in the range 2.5-40 $\mathrm{W}(0.25-4 \mathrm{~mJ}$ pulses for $10 \mathrm{kHz}$ pulsed pumping).

Further refinements are possible such as including the full bandwidth of the seed. However the results here already provide clear evidence of the benefits of pulsed pumping.

\section{RESULTS}

We first showed that for pump powers above $\sim 20 \mathrm{~W}$, the extracted pulse energy reaches a plateau at $\sim 2$ mJ. Figure 2 a) shows the variation of output power at the signal wavelength as a function of pump power in the case of c.w. pumping (square) and pulsed pumping (diamond) with the same average power and for a fiber length of $2 \mathrm{~m}$. Pulsed pumping makes a negligible difference to the maximum achievable pulse energy. The straight section of the plot shows a slope efficiency of $96 \%$, a little lower than the quantum defect between pump and signal (97.5\%). Most of this difference is due to background losses. The largest signal powers achieved correspond to pulse energies of $2.0 \mathrm{~mJ}$, i.e. $15 \mathrm{x}$ the fiber saturation energy and they occur for pump powers of approximately $20 \mathrm{~W}$.
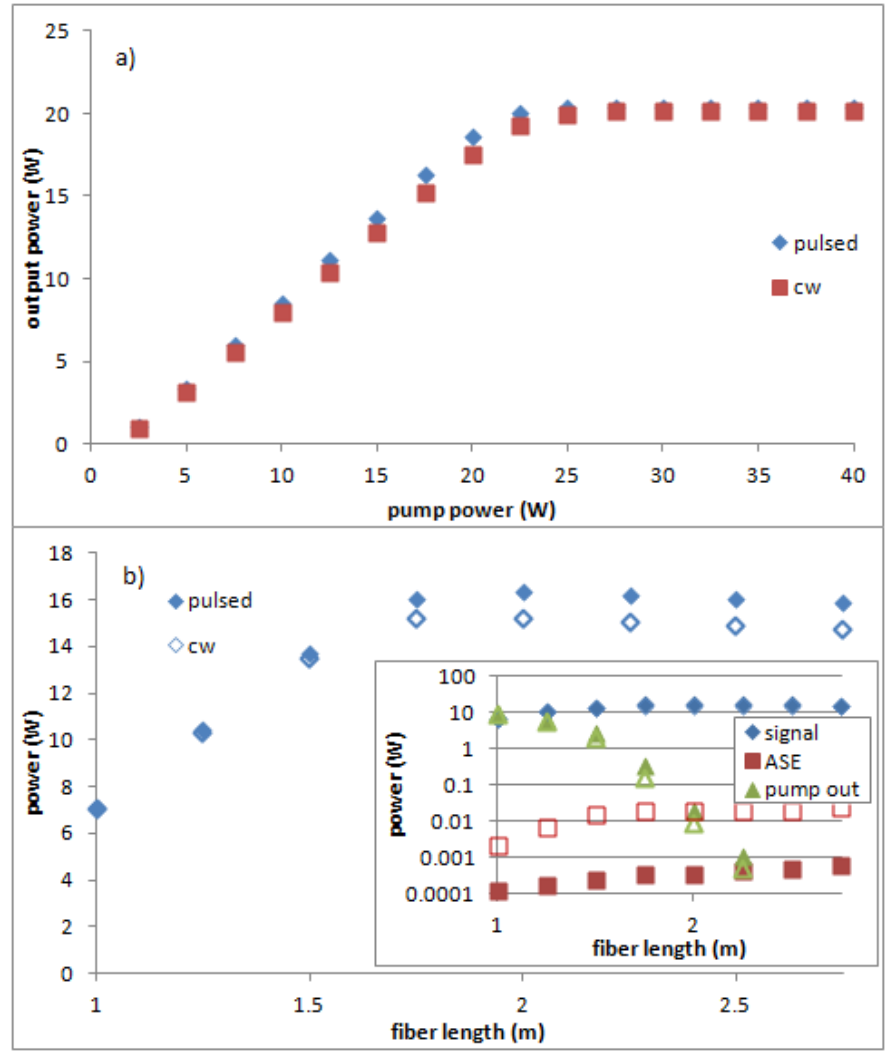

Fig. 2 a) Signal power as a function of pump power for c.w. pumping and for pulsed pumping in a $3 \mathrm{~m}$ fiber, b) signal power as a function of fiber length for $17.5 \mathrm{~W}$ pump(inset: Power on log scale to show clearly ASE and unabsorbed pump), for pulsed (filled) and c.w. (open) pumping.

We then showed that for a fixed pump power of $17.5 \mathrm{~W}$ increasing the fiber length beyond $2 \mathrm{~m}$ does not lead to significantly increased output pulse energy. Figure 2 b) shows the effect of varying the length of the fiber up to lengths of $2.75 \mathrm{~m}$ with results plotted on a linear scale in the main graph and on a log scale in the inset. The $2 \mathrm{~m}$ length absorbs the majority of the pump and enables the maximum pulse energy to be achieved. Hence, all subsequent figures are simulations for a fiber length of $2 \mathrm{~m}$.

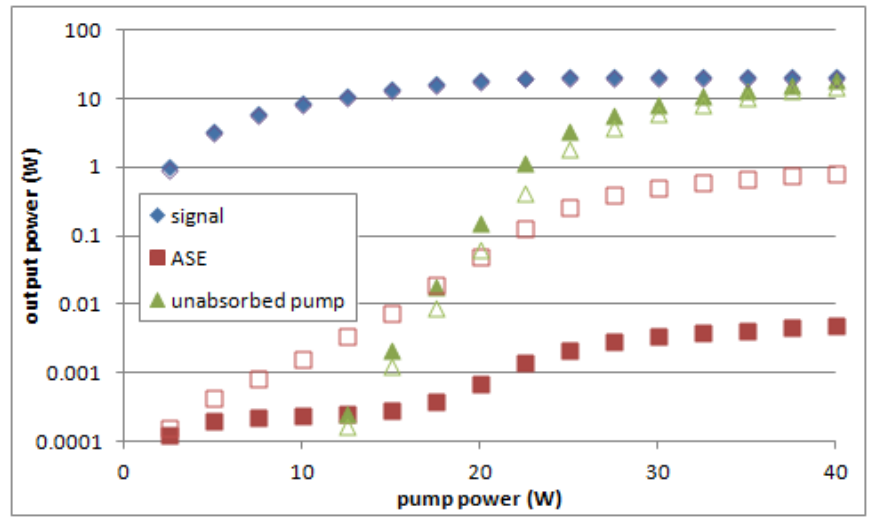

Fig. 3 The average powers of the amplified signal, ASE and unabsorbed pump at the output of the amplifier fiber as a function of input pump power for pulsed (filled) and c.w. (open) pumping.

The signal, ASE and unabsorbed pump at the output of the fiber are shown in Fig. 3, in this case using a log-scale for the y-axis. It can be seen that the ASE level and unabsorbed pump become 
significant components of the total output power at about the same pump power level. The key difference between pulsed and c.w. pumping results is that pulsed pumping produces lower levels of ASE.

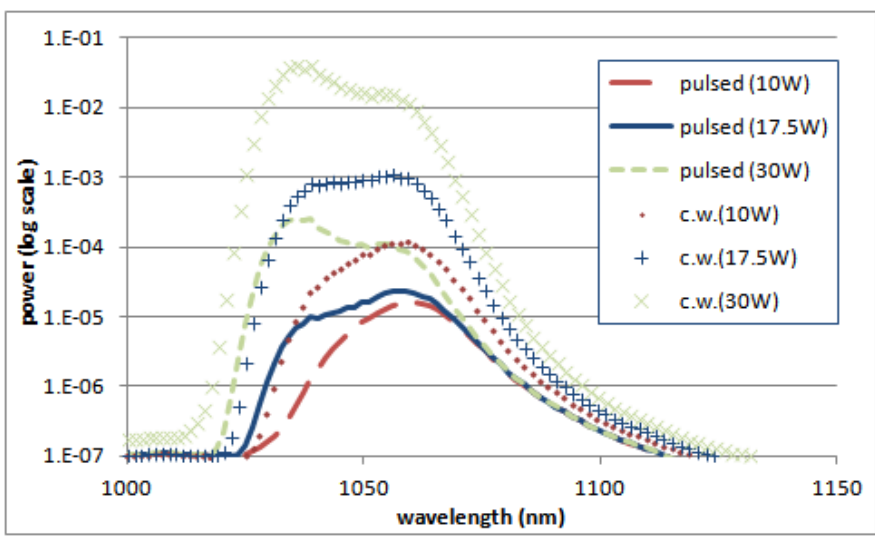

Fig. 4 ASE spectra at several pump powers, for pulsed (lines) and c.w. (symbols) pumping. For clarity, the integrated signal and pump output powers are omitted.

Figure 4 shows the output ASE spectrum at several pump powers. As expected, the ASE spectral peak is shifted to shorter wavelengths at higher powers. At low powers, the short wavelengths are reabsorbed at the output of the fiber because the inversion there is not bleached by the pump. The ASE spectrum also gives an approximate indication of the spectral region of the gain peak and confirms that our choice of 1045 $\mathrm{nm}$ for the signal is reasonable.

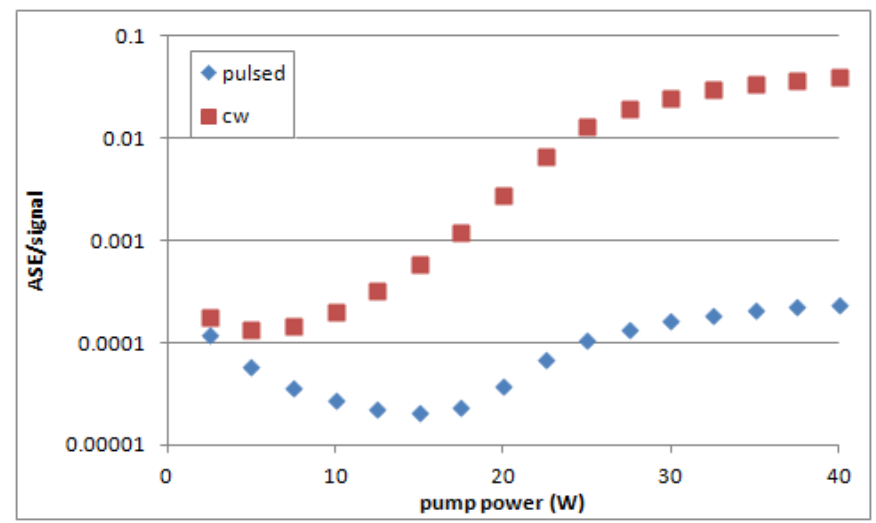

Fig. 5 Ratio of ASE to signal power vs. pump power for pulsed (diamonds) and c.w. (squares) pumping

Figure 5 replots the signal and ASE data from Fig. 3 to show the ratio of ASE to signal at the output of the fiber as a function of the average pump power for the case of c.w. pumping (squares) and pulsed pumping (diamonds). It can be seen that the use of pulsed pumping reduces the fraction of ASE in the output over a wide range of pump powers. For a pulsed pump power of $17.5 \mathrm{~W}$, the output pulse energy was $1.5 \mathrm{~mJ}$ $\left(\mathrm{E} / \mathrm{E}_{\mathrm{sat}} \sim 12\right)$ and the ASE level is reduced by approximately 2 orders of magnitude to only $2 \times 10^{-5}$ times signal power.
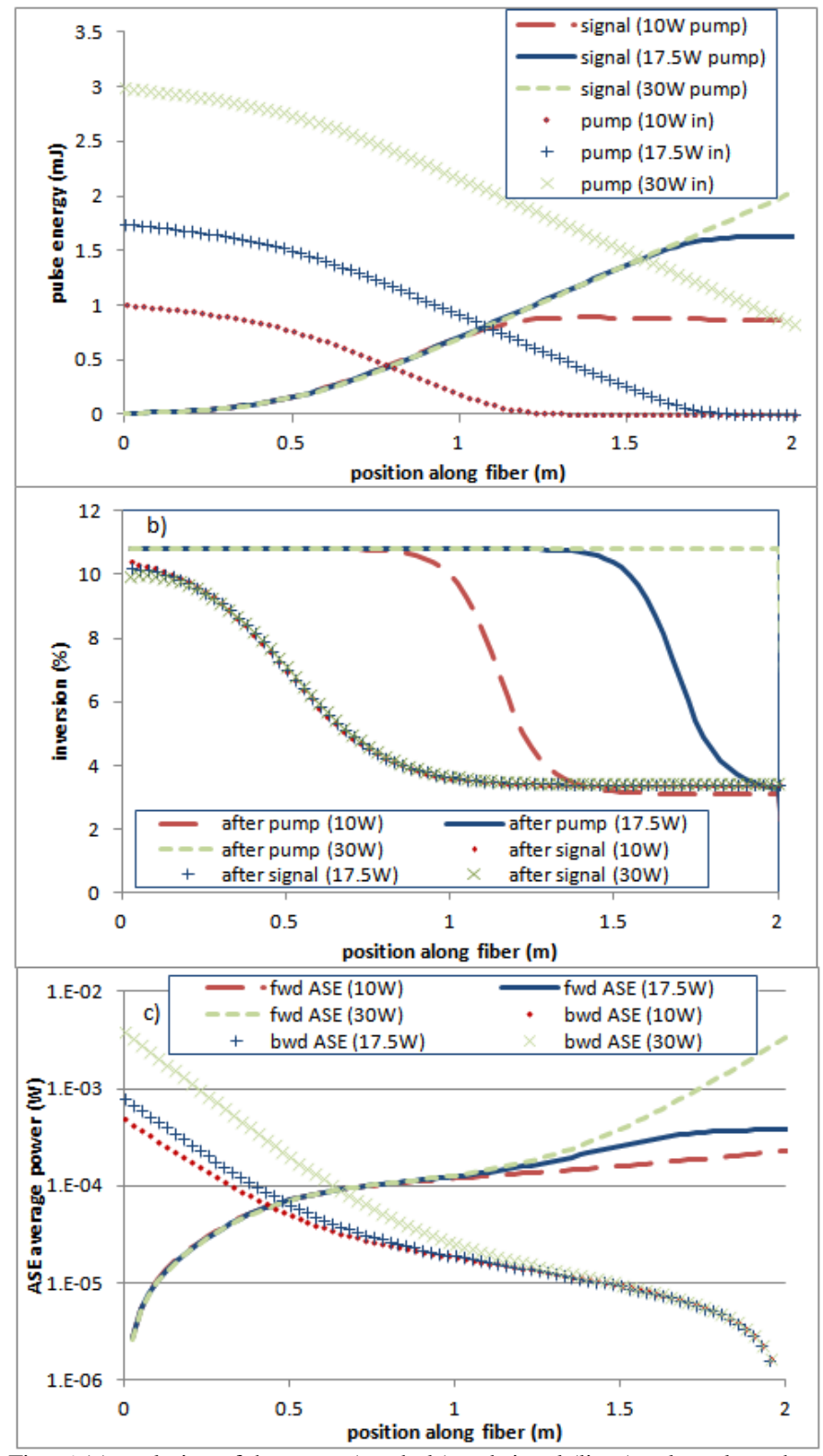

Fig. 6 (a) evolution of the pump (symbols) and signal (lines) pulses along the amplifier fiber, b) inversion in the fiber immediately after the pump pulse (solid lines) and immediately after the signal pulse (dashed lines), c) forward (solid) and backward (dashed) time-averaged ASE powers along the fiber.

Figure 6 shows evolution along the fiber for the pump and signal pulse energy, the inversion fraction and for the forwardand backward- propagating ASE. Figure 6(a) shows the evolution of the pulsed pump (symbols) and signal (lines) pulses at several power levels. Figure 6(b) shows the inversion in the fiber immediately after the pump pulse and thus before the signal extracts energy (lines) and then immediately after the signal pulse (symbols) for the same pump powers. Figure 6(c) shows the corresponding forward (lines) and backward (symbols) time-averaged ASE powers along the fiber (note the log scale on the y axis). It can be seen that the amplifier operates in the pump saturation regime, such that the pump pulse propagates further along the fiber for increased pump powers. The $\sim 4 \%$ inversion remaining after the signal pulse is the level associated with the saturation inversion at the signal wavelength. The maximum achievable pulse energy occurs 
when the pump is depleted just as it reaches the end of the fiber, and the inversion is saturated. For all three pump powers the inversion profile after the signal pulse has passed is very similar, whereas it is seen that the ASE is much higher for higher pump powers. This shows there is a rapid build-up of ASE in the 70 ns time interval between the pump creating high inversion and the signal pulses as it is in this time that the inversion and gain are significantly different for increased pump powers.

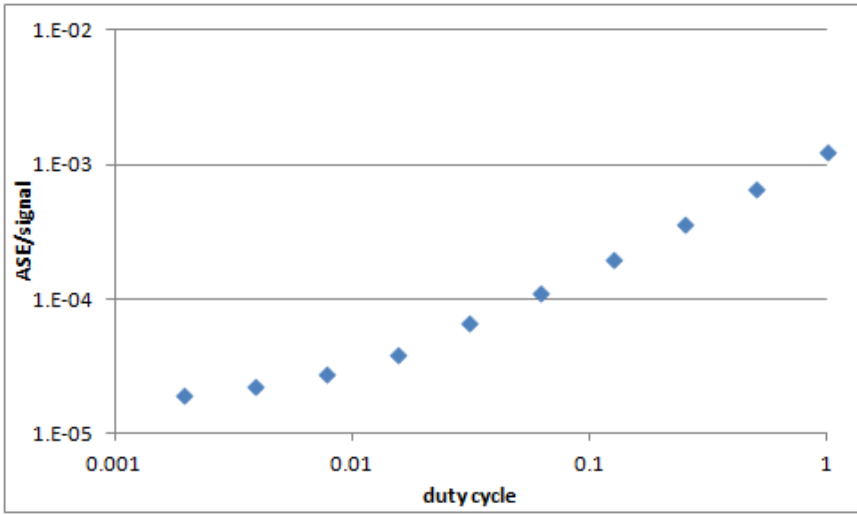

Fig. 7 ASE to signal ratio at $17.5 \mathrm{~W}$ average pump power as a function of the duty cycle of the pump pulse. (Lowest duty-cycle corresponds to $200 \mathrm{~ns}$ pump pulses and a duty-cycle of 1 corresponds to c.w. pumping.)

Figure 7 shows the effect of varying the pump pulse length from $200 \mathrm{~ns}$ to c.w. (duty cycle: $0.2 \%$ to $100 \%$ ) on the level of ASE. A constant $17.5 \mathrm{~W}$ pump average power was used and in all cases (except c.w.) the pump pulse terminates 70 ns before the peak of the input signal pulse. The variation in ASE is explained by the fact that pulsed pumping reduces the fraction of time for which inversion is high. The suppression of ASE is sufficient to permit slightly higher inversion, leading to the increase in achievable pulse energy.

\section{Sensitivity Of The Results To The Parameter VALUES IN THE MODEL}

Based on the above results, there is a clear trend showing reduced ASE as a fraction of signal when the pump duty-cycle is reduced. We considered a $10 \mathrm{kHz}$ rep. rate for the signal but that is not a fundamental requirement and the technique could be applied in systems varying from pulse on demand to $100 \mathrm{kHz}$ repetition rates. (At sub-kHz repetition rates pulsed diodepumping would probably be required for the $1018 \mathrm{~nm}$ pump.)

When considering the robustness of the conclusions we note that the simulations presented assume no additional ASE contributed by the seed or pump sources, whereas, in practice, the output ASE will be strongly dependent on the quality of these sources, and steps such as time-gating between amplifiers might be required to achieve high performance. However, further simulations (not shown) confirmed that the main result obtained, that output ASE is suppressed by up to $20 \mathrm{~dB}$ compared to c.w. pumping, remains true regardless of the level of input ASE. Increasing the number of channels or the wavelength range covered did not significantly affect the results obtained. Similar results are obtained for a counter-pumped system, but we do not have space to discuss this in the current publication. We found that the shape of the pulse does not have a significant influence on the results obtained for output power or for ASE levels. The duration of the signal pulse and the delay between the end of the pump pulse and the signal pulse can be varied without significantly affecting the output as long as they are short compared to the pump pulse duration. We observed that varying the pump pulse shape has only a very minor effect on the dynamics compared to the effect of changing the pulse duration.

We have considered Yb-fiber pumping Yb-fiber, whereas other possibilities exist such as using a Nd-doped fiber to pump the Yb fiber. In summary, our simulations show that pulsed pumping achieves a reduction in ASE level of $\sim 2$ orders of magnitude for pulsed tandem pumping compared to tandem pumping with a c.w. source. These conclusions are not significantly changed by details of the pump pulse shape, or the exact value of backscattering coefficients.

Another consideration for the final amplifier is the $\mathrm{Yb}$ doping concentration. Higher concentration may be permissible if photo-darkening is indeed reduced and this would enable more rapid pump absorption and hence shorter device lengths to further reduce nonlinear effects. In practice the thermal load per unit length will also be important and using moderate doping levels comparable to those used in currently available commercial fibers may be appropriate.

As the saturation fluence, nonlinear effects and pump absorption scale linearly with the mode area, $\mathrm{A}_{\text {eff }}$ (for fibers of the same core glass composition) we expect the choice of a shorter rod-fiber would mean that the limiting factor becomes optical damage at below the maximum possible extractable pulse energies from a gain-storage perspective. However, a full comparison of the performance of tandem pumped vs. $975 \mathrm{~nm}$ c.w. diode pumped rod-fibers to include nonlinearity and optical damage is beyond the scope of this paper.

\section{DISCUSSION}

The rate of pump absorption in an amplifier is proportional to $\left(\left(\sigma_{\mathrm{e}}\left(\lambda_{\text {pump }}\right)-\sigma_{\mathrm{a}}\left(\lambda_{\text {pump }}\right) \mathrm{N}_{2}-\sigma_{\mathrm{a}}\left(\lambda_{\text {pump }}\right)\right) \Gamma_{\text {pump }}\right.$, where $\Gamma_{\text {pump }}$ is the overlap fraction for the pump and the doped core, $\sigma_{e, a}\left(\lambda_{\text {pump }}\right)$ are the pump emission and absorption cross sections and $\mathrm{N}_{2}$ is the fractional inversion. For a commercially available $\mathrm{Yb}$ fiber with a rod-type geometry having clad diameter of $260 \mu \mathrm{m}$ and doped core diameter of $50 \mu \mathrm{m}$ (NKT Photonics aeroGAIN-RODPM85) inversion is low enough to ignore the inversion dependent term and the product - $\sigma_{\mathrm{a}}\left(\lambda_{\text {pump }}\right) \Gamma_{\text {pump }}$ is $\sim 7 \mathrm{x} 10^{-26} \mathrm{~m}^{2}$; for a tandem core-pumped fiber with $1018 \mathrm{~nm}$ pumping and assuming a pump overlap of 0.9 this product is $4.5 \times 10^{-26} \mathrm{~m}^{2}$. In practice, for the tandem-pumped system, the inversion dependent term is significant at the pump input end of the fiber. In the simulations described above we saw that the pump absorption varied from being consistent with the value above at the output end to being more than an order of magnitude lower at the pump input. For the power levels we were considering this made the optimum fiber length about a factor of 2-3 longer than might be predicted from the simple comparison above. 
Therefore the required fiber length is of order 4 times greater for the system described than for a Rod with equivalent doping concentration. Whilst the increased length is a potential disadvantage due to increased nonlinear effects, it is potentially outweighed in many circumstances by the other advantages of this system.

Tandem pumping has the advantage of reduced quantum defect heating when used with the $1018 \mathrm{~nm}$ pump and $1045 \mathrm{~nm}$ signal considered above compared to $975 \mathrm{~nm}$ pumped rod-type fibers operated close to $1030 \mathrm{~nm}$.

Other examples of three-level lasers where tandem pumping has been applied is the use of Thulium fiber lasers to pump Holmium lasers in the $2 \mu \mathrm{m}$ wavelength range [25]]. The only experimental examples of pulsed resonant pumping we are aware of involve pulsed pumping of Holmium [26, 27] or Thulium [28-30] gain-switched fiber lasers $(1940 \mathrm{~nm})$ in order to regulate the usually chaotic pulse generation, and the average powers have been at the $<8$-W level.

Finally, the application of pulsed tandem pumping will be for short pulse systems where the extractable energy is limited not by the ability to store the maximum possible energy, but the rather lower energy at which the onset of nonlinear effects become significant. At higher powers damage can also be important [31].

For $\sim 10$ ps pulses SPM is less of a problem than for femtosecond systems because a distortion from SPM that creates e.g. 1 ps of additional pulse broadening does not significantly change the characteristics of a 10 ps pulse whereas it drastically changes a 200 fs pulse. For fs systems, rod-type systems with pulse shaping setups that combine chirped-pulseamplification (CPA) [32] and DPA may be needed. We do not consider fs pulses in detail here, but the benefits obtained in the ps case will still apply in general terms.

The cost and complexity of a single tandem-pumped amplifier is likely to be greater than a diode-pumped fiber version but this may be outweighed by the energy storage in the pump laser which offers some technical performance advantages as shown below. Some areas where this cost may be justified are where the performance of the preferred $975 \mathrm{~nm}$ diode-pumped rod-type fibers is limited by thermally induced modal instability [1,33-35] and where the application requires repetition rate and average power scaling beyond this limit, such as for space-debris management [11]. The references show that in a $200 \mathrm{~W}$ class rod-type fiber amplifier, approximately equal heat contributions come from quantum defect heating and from photo-darkened $\mathrm{Yb}$ ions that absorb pump light without providing laser gain. The references additionally makes a close link between the heat load per unit length and the onset of thermally induced modal instability. In a pulsed pumped tandem amplifier, as considered above, the quantum defect is lower than for a $975 \mathrm{~nm}$ pumped rod-fiber with signal wavelength at $1030 \mathrm{~nm}$ due to the relative proximity of pump and signal wavelengths in the two systems. Therefore we would expect the heat-related output power threshold for thermally induced modal instability to be reached at a higher output power in the tandem pumped system above than is the case for a $975 \mathrm{~nm}$ pumped rod-type fiber. (Any photodarkening heat load depends in detail on both the glass composition of the gain medium and on the fraction of excited Yb-ions [36, 37] and therefore it is not possible to compare the heating from this effect without knowing the precise glass compositions and operating conditions selected for a given application). In such a large project the additional cost of the amplifier may be more than compensated by reduced facilities costs (cooling/controlsystems) and more stable operation because the tandempumped fibers should have alignment-free fiberized pumps and should be more resistant to photo-darkening.

\section{CONCLUSION}

In conclusion, sub- $\mu$ s pulsed tandem pumping offers a relatively straightforward way to reduce the ASE and hence improve the performance of $\mathrm{kHz}$ or lower repetition rate fiber amplifiers. The in-band pump amplifier enables conversion of the low-brightness pump diodes into a high-brightness single / few -mode output for core pumping the final amplifier. This keeps the final amplifier short despite the reduced absorption cross sections compared to the absorption peak and ensures the technique is compatible with ultra-short pulse operation. The in-band pump amplifier also enables energy storage to create the required sub- $\mu$ s pump pulses. This is a new regime and quite different from pulsed diode pumping that has traditionally been used for low-repetition rate fiber lasers in that the diodes do not store energy.

The attractions of c.w. tandem pumping for $\mathrm{Yb}$ amplifiers have already motivated the development of high power shortwavelength pump fiber lasers which are needed to keep the length of the tandem amplifier short [38-40]. The sub- $\mu$ s pulse pumping technique outlined here could further encourage experimental research in the field.

We thank Prof. J Nilsson and Prof. Sir D.N. Payne for their encouragement of this research and for useful discussions.

\section{REFERENCES}

M. N. Zervas and C. A. Codemard, "High Power Fiber Lasers: A Review," Selected Topics in Quantum Electronics, IEEE Journal of, vol. 20, pp. 219-241, Sep-Oct 2014.

[2] G. Mourou, B. Brocklesby, T. Tajima, and J. Limpert, "The future is fibre accelerators," Nat Photon, vol. 7, pp. 258-261, 2013.

[3] W. S. Brocklesby, J. Nilsson, T. Schreiber, J. Limpert, A. Brignon, J. Bourderionnet, et al., "ICAN as a new laser paradigm for high energy, high average power femtosecond pulses," The European Physical Journal Special Topics, vol. 223, pp. 1189-1195, 1 May 2014.

[4] J. D. Minelly, R. I. Laming, J. E. Townsend, W. L. Barnes, K. P. Jedrzejewski, and D. N. Payne, "High-gain fibre power amplifier tandem-pumped by a $3 \mathrm{~W}$ multi-stripe diode," presented at the OFC'92: Conference on Optical Fibre Communications (proceedings pg. 32-33), 1992.

[5] J. Zhu, P. Zhou, Y. Ma, X. Xu, and Z. Liu, "Power scaling analysis of tandem-pumped Yb-doped fiber lasers and amplifiers," Optics Express, vol. 19, pp. 18645-18654, 12 Sept. 2011.

[6] Y. Zhu, P. Zhou, H. Zhang, X. Wang, and S. Guo, "Analysis of the power scaling of resonantly pumped Tm-doped silica fiber lasers," in International Symposium on Photoelectronic Detection and Imaging: High Power Lasers and Applications, , 2013, pp. 89040R89040R-8.

[7] Y. M. Chang, T. Yao, H. Jeong, J. Ji, S. Yoo, T. C. May-Smith, et al., "3 \% Thermal Load Measured in Tandem-pumped Ytterbium- 
doped Fiber Amplifier," in CLEO: 2014, San Jose, California, 2014, p. STh4N.7.

[8] A. Ferin, V. Gapontsev, V. Fomin, A. Abramov, M. Abramov, and D. Mochalov, "17 kW CW laser with $50 \mu \mathrm{m}$ delivery," in 6th International Symposium on High-Power Fiber Lasers and Their Applications, St Petersburg, 2012.

[9] E. Shcherbakov, V. Fomin, A. Abramov, A. Ferin, D. Mochalov, and V. P. Gapontsev, "Industrial Grade $100 \mathrm{~kW}$ Power CW Fiber Laser," in Advanced Solid-State Lasers Congress, Paris, France, 2013, p. ATh4A.2.

[10] C. R. Giles and E. Desurvire, "Modeling Erbium-Doped Fiber Amplifiers," Journal of Lightwave Technology, vol. 9, pp. 271-283, Feb 1991.

[11] R. Soulard, M. N. Quinn, T. Tajima, and G. Mourou, "ICAN: A novel laser architecture for space debris removal," Acta Astronautica, vol. 105, pp. 192-200, December 2014.

[12] R. Paschotta, J. Nilsson, A. C. Tropper, and D. C. Hanna, "Ytterbium-doped fiber amplifiers," IEEE Journal of Quantum Electronics, vol. 33, pp. 1049-1056, Jul 1997.

[13] K. W. Morton and D. F. Mayers, Numerical solution of partial differential equations: an introduction, 2nd ed.: Cambridge University Press, 2005.

[14] S. Zhou, F. W. Wise, and D. G. Ouzounov, "Divided-pulse amplification of ultrashort pulses," Optics Letters, vol. 32, pp. 871873, 1 April 2007.

[15] M. Lapczyna, K. P. Chen, P. R. Herman, H. W. Tan, and R. S. Marjoribanks, "Ultra high repetition rate (133 MHz) laser ablation of aluminum with 1.2-ps pulses," Applied Physics A, vol. 69, pp. S883-S886, 01 December 1999.

[16] I. Will, H. I. Templin, S. Schreiber, and W. Sandner, "Photoinjector drive laser of the FLASH FEL," Optics Express, vol. 19, pp. 2377023781, 21 Nov. 2011.

[17] P. Elahi, S. Yılmaz, Y. B. Eldeniz, and F. Ö. Ilday, "Generation of picosecond pulses directly from a $100 \mathrm{~W}$, burst-mode, dopingmanaged Yb-doped fiber amplifier," Optics Letters, vol. 39, pp. 236-239, 15 Jan. 2014.

[18] D. N. Schimpf, C. Ruchert, D. Nodop, J. Limpert, A. Tünnermann, and F. Salin, "Compensation of pulse-distortion in saturated laser amplifiers," Optics Express, vol. 16, pp. 17637-17646, 27 Oct. 2008.

[19] A. Malinowski, K. T. Vu, K. K. Chen, J. Nilsson, Y. Jeong, S. Alam, et al., "High power pulsed fiber MOPA system incorporating electro-optic modulator based adaptive pulse shaping," Optics Express, vol. 17, pp. 20927-20937, 9 Nov. 2009.

[20] R. G. Smith, "Optical power handling capacity of low loss optical fibers as determined by stimulated Raman and Brillouin scattering," Applied Optics, vol. 11, pp. 2489 - 2494, 1972.

[21] A. H. Hartog and M. P. Gold, "On the theory of backscattering in single-mode optical fibers," Journal of Lightwave Technology, vol. 2, pp. 76-82, 1984.

[22] H. M. Pask, R. J. Carman, D. C. Hanna, A. C. Tropper, C. J. Mackechnie, P. R. Barber, et al., "Ytterbium-Doped Silica Fiber Lasers - Versatile Sources for the 1-1.2 micron Region," IEEE Journal of Selected Topics in Quantum Electronics, vol. 1, pp. 2-13, Apr 1995.

[23] M. N. Zervas and R. Laming, "Rayleigh scattering effect on the gain efficiency and noise of erbium-doped fiber amplifiers," Quantum Electronics, IEEE Journal of, vol. 31, pp. 468-471, 1995.

[24] P. Mazumder, S. L. Logunov, and S. Raghavan, "Analysis of excess scattering in optical fibers," Journal of Applied Physics, vol. 96, pp. 4042-4049, 2004.

[25] A. Hemming, S. Bennetts, N. Simakov, A. Davidson, J. Haub, and A. Carter, "High power operation of cladding pumped holmiumdoped silica fibre lasers," Optics Express, vol. 21, pp. 4560-4566, 25 Feb. 2013.

[26] S. Hollitt, N. Simakov, A. Hemming, J. Haub, and A. Carter, "A linearly polarised, pulsed Ho-doped fiber laser," Optics Express, vol. 20, pp. 16285-16290, 16 July 2012.

[27] K. S. Wu, D. Ottaway, J. Munch, D. G. Lancaster, S. Bennetts, and S. D. Jackson, "Gain-switched Holmium-doped fibre laser," Optics Express, vol. 17, pp. 20872-20877, 09 November 2009.

[28] T. Yulong, L. Feng, and X. Jianqiu, "High Peak-Power GainSwitched $\mathrm{Tm}^{3+}$-Doped Fiber Laser," Photonics Technology Letters, IEEE, vol. 23, pp. 893-895, 2011.
[29] M. Jiang and P. Tayebati, "Stable $10 \mathrm{ns,} \mathrm{kilowatt} \mathrm{peak-power} \mathrm{pulse}$ generation from a gain-switched Tm-doped fiber laser," Optics Letters, vol. 32, pp. 1797-1799, 1st July 2007.

[30] N. Simakov, A. Hemming, S. Bennetts, and J. Haub, "Efficient, polarised, gain-switched operation of a Tm-doped fibre laser," Optics Express, vol. 19, pp. 14949-14954, 1st Aug. 2011.

[31] J. W. Dawson, M. J. Messerly, R. J. Beach, M. Y. Shverdin, E. A. Stappaerts, A. K. Sridharan, et al., "Analysis of the scalability of diffraction-limited fiber lasers and amplifiers to high average power," Optics Express, vol. 16, pp. 13240-13266, 18 Aug. 2008.

[32] D. Strickland and G. Mourou, "Compression of Amplified Chirped Optical Pulses," Optics Communications, vol. 56, pp. 219-221, Dec 11985.

[33] C. Jauregui, H.-J. Otto, F. Stutzki, J. Limpert, and A. Tünnermann, "Simplified modelling the mode instability threshold of high power fiber amplifiers in the presence of photodarkening," Optics Express, vol. 23, pp. 20203-20218, 10 Aug. 2015.

[34] C. Jauregui, T. Eidam, J. Limpert, and A. Tünnermann, "Impact of modal interference on the beam quality of high-power fiber amplifiers," Optics Express, vol. 19, pp. 3258-3271, 14 Feb. 2011.

[35] A. V. Smith and J. J. Smith, "Mode instability in high power fiber amplifiers," Optics Express, vol. 19, pp. 10180-10192, 23 May 2011.

[36] M. N. Zervas, F. Ghiringhelli, M. K. Durkin, and I. Crowe, "Distribution of photodarkening-induced loss in Yb-doped fiber amplifiers," in SPIE-Photonics West, 2011, pp. 79140L-79140L-8.

[37] S. Taccheo, H. Gebavi, A. Monteville, O. Le Goffic, D. Landais, D. Mechin, et al., "Concentration dependence and self-similarity of photodarkening losses induced in Yb-doped fibers by comparable excitation," Optics Express, vol. 19, pp. 19340-19345, 26 Sept. 2011.

[38] F. Beier, H. J. Otto, C. Jauregui, O. de Vries, T. Schreiber, J. Limpert, et al., "1009 nm continuous-wave Ytterbium-doped fiber amplifier emitting 146 W," Optics Letters, vol. 39, pp. 3725-3727, 01 July 2014.

[39] G. Gu, Z. Liu, F. Kong, H. Tam, R. K. Shori, and L. Dong, "Highly efficient ytterbium-doped phosphosilicate fiber lasers operating below 1020nm," Optics Express, vol. 23, pp. 17693-17700, 13 July 2015.

[40] H. Xiao, P. Zhou, X. L. Wang, X. J. Xu, and Z. J. Liu, "High power $1018 \mathrm{~nm}$ ytterbium doped fiber laser with an output power of 309 W," Laser Physics Letters, vol. 10, p. 065102, 2013.

Dr. Andrew Malinowski received a BA in Natural Sciences from the University of Cambridge in 1992 and a $\mathrm{PhD}$ in Physics from the University of Southampton in 1996.

He has worked at the University of Sheffield (1996-97) and the University of Southampton (1997-2011). From 2001 he worked at the Optoelectronics Research Centre, University of Southampton on fiber laser and amplifier systems and their applications. Since 2011 he has worked at SPI Lasers, Southampton, UK, on fiber laser development.

Dr. Jonathan H. V. Price received the Ph.D. degree in optoelectronics from the Optoelectronics Research Centre, University of Southampton, U.K., in 2003.

He then held a Royal Academy of Engineering Postdoctoral Research Fellowship at the Optoelectronics Research Centre, University of Southampton and has continued working in his main research areas of novel microstructured optical fibers for 
ultrashort-pulse applications and the development of highpower femtosecond pulsed fiber laser sources.

Prof. Michalis N. Zervas graduated from the Electrical Engineering Department, University of Thessaloniki, Thessaloniki, Greece, in 1984. He received the M.Sc. degree in applied and modern optics (with distinction) from the University of Reading, Reading, U.K., in 1985 and the Ph.D. degree in fiber optics from University College London, London, UK., in 1989.

He joined the Optoelectronics Research Centre, University of Southampton, Southampton, U.K., in 1991 as a Research Fellow and was promoted to Research Lecturer in 1995 and Professor in Optical Communications in 1999. His research activities include advanced optical fiber amplifier configurations, high-power fiber lasers and applications, fiber distributed feedback (DFB) lasers, Bragg grating theory and devices, surface-plasmon effects and devices, optical microresonators, and nonlinear fiber optics. He is a cofounder of Southampton Photonics Inc., a University of Southampton spinoff manufacturing high-power fiber lasers, where he is currently serving as Chief Scientist.

He has authored/coauthored over 300 technical publications, about 40 patents/patent applications, of which 20 are granted, and has served on various conference program committees. Dr. Zervas was the General Program Co-Chair of the 1999 Optical Society of America (OSA) Optical Amplifiers Meeting. He has given a number of invited talks and short courses in fiber amplifiers and fiber Bragg gratings at major international conferences. He was a co-editor of the journal Integrated and Fiber Optics, special issue on fiber Bragg gratings. In 1996, he shared the prestigious Metrology award from the Confederation of British Industry for his work on grating measuring systems for characterizing reflection and dispersion performance of fiber Bragg gratings. In 2006, he was finalist for the Royal Society of Engineering McRobert Award for the development of high-power industrial fiber lasers. He was elected OSA Fellow in 2015. 